\title{
Environmental factors influence the innate stimulatory capacity of quinoa proteins
}

\author{
Victor Zevallos ${ }^{1}$ and Irene Herencia ${ }^{2}$ \\ ${ }^{1}$ St Mary's University, London, United Kingdom and \\ ${ }^{2}$ Universidad Politécnica de Madrid, Madrid, Spain
}

\section{Abstract}

Plant proteins constitute a rich and complex source of nutrients with health benefits for most populations. However, an ever-growing number of people have been affected by protein allergies and sensitivities. Furthermore, there is evidence suggesting that plant proteins can activate the innate immune system by stimulating the toll like receptor 4 (TLR4)-MD2-CD14 complex in cells of the mononuclear phagocyte system. Therefore, we have explored the effects of genetic variability, agricultural practices and area of cultivation (Andes vs Mediterranean) on the innate stimulatory capacity of quinoa proteins.

Plant proteins were exhaustively extracted from milled and defatted samples using salt solutions or enzymatic digestion (pepsin/ trypsin) and dialyzed against neutral buffers, sterile filtrated and lyophilized. Ethanol soluble proteins were extracted from the resulting pellet and additionally, milled samples were digested with pepsin and trypsin, dialyzed and sterile filtrated. Extracts were cultured with monocytic cell lines and the concentration of IL-8 and other innate cytokines released was measured in cell culture supernatants after overnight incubation. Additionally, samples were tested with cells transfected with TLR4-MD2-CD14 and the Renilla luciferase reporter gene under the transcriptional control of the IL-8 promoter.

We observed that proteins from 4 quinoa cultivars from the Andes (cultivated at 3800 meters above sea level) had very low innate immune stimulatory activity $(<5 \%$ of modern wheat). However the same quinoa variants cultivated at sea level had significantly higher bioactivity ( $>10 \%$ higher than modern wheat) which was absent in the ethanol extracts and reduced by $\sim 60 \%$ in the enzymatically digested extracts. Other samples such as millet and buckwheat also showed reduced bioactivity compared to modern wheat after enzymatic digestion in murine and human cells. To conclude: 1) We have identified that the innate stimulatory activity of quinoa proteins is significantly affected by agricultural practices and area of cultivation; 2) This immune-stimulatory activity can be reduced by enzymatic digestion; 3) Depending on the varieties, cultivation and further processing, plant proteins may impact on the development of allergies and sensitivities.

\section{Conflict of Interest}

There is no conflict of interest 